\title{
Towards a conceptualization of sociomaterial entanglement ${ }^{\star}$
}

\author{
Roberta Ferrario and Daniele Porello ${ }^{1}$ \\ Institute of Cognitive Sciences and Technologies, CNR \\ \{daniele.porello, roberta.ferrario\}@loa.istc.cnr.it
}

\begin{abstract}
In knowledge representation, socio-technical systems can be modeled as multiagent systems in which the local knowledge of each individual agent can be seen as a context. In this paper we propose formal ontologies as a means to describe the assumptions driving the construction of contexts as local theories and to enable interoperability among them. In particular, we present two alternative conceptualizations of the notion of sociomateriality (and entanglement), which is central in the recent debates on socio-technical systems in the social sciences, namely critical and agential realism.

We thus start by providing a model of entanglement according to the critical realist view, representing it as a property of objects that are essentially dependent on different modules of an already given ontology. We refine then our treatment by proposing a taxonomy of sociomaterial entanglements that distinguishes between ontological and epistemological entanglement. In the final section, we discuss the second perspective, which is more challenging form the point of view of knowledge representation, and we show that the very distinction of information into modules can be at least in principle built out of the assumption of an entangled reality.
\end{abstract}

\section{Introduction}

Starting from the 60's, scholars began to use the concept of socio-technical system [11] to describe and analyze workplaces and wider infrastructures in which the technological component played a pivotal role in the production system, but it could also be turned into a threat to the very same system and to its participants, due to its interlacement with the human dimension of work. Roughly speaking, such approaches and those taking inspiration from them see a socio-technical system as a hybrid system, constituted by interacting components that are heterogeneous and which, in order to be analyzed, require different theoretical instruments, those of the social and natural sciences.

With the continuous advances in science and technologies, some devices inhabiting socio-technical systems have been endowed with functionalities that are so complex and effective that their behavior can be more and more rightly dubbed as "intelligent" and "autonomous". In fact, already in the 1990's, scholars in distributed artificial intelligence started to use the multiagent paradigm [8] [17] to model socio-technical systems and nowadays intelligent agents play an important role in many work environments.

\footnotetext{
* Supported by the VisCoSo project grant, financed by the Autonomous Province of Trento through the "Team 2011" funding programme.
} 
In the artificial intelligence community, and especially scholars embracing the multiagent approach, in order to represent (among other things) the knowledge and perspective of agents [16] have been using contexts, intended in rough terms as local theories that the agents entertain with respect to the domain of interest. The contextual approach has shown to be very useful in representing both the "local" perspective of each agent and if and how such perspectives can be integrated through the use of "lifting" [23] or compatibility [16] rules.

Ideally, each local context representing the perspective of an agent can have its own language and inference rules, and each language bears an ontological commitment with respect to how the terms of the vocabulary should be interpreted to express the agent's perspective on the domain of interest and which is the intended meaning of the terms that are used in the local language.

The idea of using formal ontologies to constrain the interpretation of the local languages of artificial agents is not new [13]; in this paper we would like to show how they can be used also to express different ways in which the agents conceptualize the socio-technical systems they inhabit, starting from a specific notion that has recently been much debated, especially in sociology and organization science, that of sociomateriality and the connected notion of entanglement.

For many years, the studies on socio-technical systems have been viewing such components as interacting but sharply separated, resulting in approaches focused on the degrees of adoption of new material technologies by the operators of socio-technical systems, or on the reshaping of the organizational structures induced by the introduction of technology. Such view is in fact the one that has been widely adopted by the knowledge representation community for modeling socio-technical systems.

Nonetheless, more recently, in social and organizational studies a different reading of socio-technical systems has been spreading, more focused on work practices, which are seen as sociomaterial, where the two dimensions of sociality and materiality are so tightly intertwined that are not really separable. And it is in these studies that the concept of entanglement has been employed for the description of sociomateriality:

Such an alternative view asserts that materiality is integral to organizing, positing that the social and the material are constitutively entangled in everyday life. A position of constitutive entanglement does not privilege either humans or technology (in one-way interactions), nor does it link them through a form of mutual reciprocation (in two-way interactions). Instead, the social and the material are considered to be inextricably related - there is no social that is not also material, and no material that is not also social. [24, 1437]

These studies have succeeded in explaining, through theoretical and empirical analyses conducted in real case studies, how entanglement is enacted in organizational life. A scholar who, taking inspiration from a notion elaborated in quantum physics, developed a new conception of entanglement to be applied to social analysis is Karen Barad:

[...] the primary ontological unit is not independent objects with inherent boundaries and properties but rather phenomena. In my agential realist elaboration, phenomena do not merely mark the epistemological inseparability of 
observer and observed, or the results of measurements; rather, phenomena are the ontological inseparability/entanglement of intra-acting "agencies." That is, phenomena are ontologically primitive relations - relations without preexisting relata. $[1,139]$

In social and organization studies this idea is being applied in manyfold ways, to talk about the inseparability of social and material dimension, of the scholar (observer) and the object of his/her observation, of the actors, their agencies and the networks they belong to, etc.

However, besides Barad's agential realism, another philosophical position has been proposed as a theoretical foundation for the studies on sociomateriality, namely that of critical realism [19]. Even though both approaches hold sociomateriality as a proper lens to look at organizations, they differ in attributing a metaphysical primacy to the sociomaterial over the social and the material (agential realism) or vice versa (critical realism).

Leonardi states very clearly such difference:

The main crux of the difference in theoretical foundation offered by agential realism and critical realism is that the former treats the "sociomaterial" as something that pre-exists people's perceptions of it while the latter argues that the "social" and the "material" are independent entities that become "sociomaterial" as they are put into relationship with one another through human action.

When we come to knowledge representation and multiagent modeling, we can say that formal modeling approaches to socio-technical systems have mostly neglected the usefulness of this notion and this is quite surprising, as knowledge representation is mainly concerned with the way in which a rational agent may conceive and represent a certain domain of discourse. It is exactly from this perspective that sociomateriality is an important analytical category, either considered as the explanation of how sociotechnical systems are metaphysically constituted, or of the "imbrication" [19] of social and material resulting from agents' action within the system.

Concerning this point, we would like to stress the fact that our final aim is to build an ontological model of socio-technical systems acknowledging the importance of sociomateriality and able to represent both positions as the result of alternative ontological choices. The rationale is that, if one wants to represent how artificial agents interpret the features of socio-technical systems by local theories as contexts and then wants to compare and put them into communication or, in other words, wants these theories to be interoperable, it is necessary to make explicit the assumptions behind each one of these local theories. Formal ontologies are a powerful tool for this endeavor and, in the case of socio-technical systems, a good starting point is to make explicit which are the assumptions behind the notions of sociomateriality that the agents use. Therefore, in our framework the ontological analysis precedes and guides the modelization of the local knowledge of agents with the use of contexts by making explicit which are the most important ontological choices with respect to the domain of interest. One of such choices for socio-technical systems is how to conceptualize the relation between sociality and materiality. 
In this paper we present the proposal to formalize one of the possible interpretations of the term "entanglement", namely that of the critical realism and we leave the formalization of the alternative ontological choice, that of agential realism, for future work.

In formal ontologies, a methodology has been devised to deal with very complex models (as is the case for models of socio-technical systems), which goes under the name of "modularization". This consists in isolating meaningful fragments of an ontology, which can be used as stand-alone sub-parts of the ontology, where reasoning is facilitated by the fact of being applied only to the categories of the module and not to all the categories of the ontology. Modules are thus good candidates to represent different aspects or realms characterizing a socio-technical system, like the physical, the mental and the social. But what happens when properties belonging to different realms of information, for instance from the physical and from the normative social realm are ascribed to the same entities? We call "entanglement" the dependence of an entity from entities belonging to different realms (as the social and the material).

The first step of our proposal for capturing entanglement in knowledge representation under the critical realist view is by representing the different realms as different modules in an ontology and entanglement as the interdependency of entities belonging to different modules of an ontology. In other terms, we conceptualize entanglement in ontologies as the need, in order to characterize certain entities belonging to a certain module of an ontology, of categories belonging to different modules. Thus, entanglement becomes a feature of an object (or a concept) that exhibits an essential interdependence between different realms, such as the social and the physical, or the physical and the mental.

Furthermore, we will introduce a first classification of types of entanglement, distinguishing:

- ontological entanglement: an object that is ontologically dependent on objects in different modules (e.g. physical and social, physical and mental);

- epistemological entanglement: an object or concept such that every possible definition of the object/concept requires concepts/objects belonging to different realms.

What we will try to provide with this work, rather than a sharp classification of phenomena under one or the other type of entanglement, is a framework enabling modelers to represent various types of entanglement by choosing the type that they deem more appropriate to represent the phenomena they want to model.

The remainder of this paper is organized as follows. Section 2 introduces some fundamental features of a foundational ontology. We place our treatment within DOLCE [21] and discuss how to classify the elements of the ontology into modules (i.e. physical, social, mental module). Section 3 presents a formalization of entanglement under the critical realist view and the further distinction between ontological and epistemological entanglement and, in Section 4 we sketch some preliminary ideas for formalizing the agential realist view and we foresee the need to this aim to "turn upside down" the way in which we model in knowledge representation. 


\section{Ontological analysis: DOLCE}

We present some basic features of DOLCE-CORE, the ground ontology, to show that they allow for keeping track of the rich structure of information in a socio-technical system [26]. For an introduction to DOLCE-CORE, we refer to [4], here we simply point at the relevant features. The ground ontology is designed to be general and domain independent. This is motivated by the need of a common language to talk about very general properties that are ascribable to entities belonging to different domains.

The ontology partitions the objects of discourse, labelled particulars PT into the following six basic categories: objects $\mathrm{O}$, events $\mathrm{E}$, individual qualities $\mathrm{Q}$, regions $\mathrm{R}$, concepts $\mathrm{C}$, and arbitrary sums AS. The six categories are to be considered rigid, i.e. a particular cannot change category through time. For example, an object cannot become an event. In particular, we shall focus on the following categories.

Objects represent particulars that are mainly located in space, e.g. a screwdriver. On the other hand, events have properties that are mainly related to time, e.g. , the boarding of flight 717. The relation that links objects and events is the participation relation: "an object $x$ participates in an event $y$ at time $t$ " PC $(x, y, t)$.

An individual quality is simply an entity that we can perceive and measure, which inheres to a particular (e.g. the weight of a hammer, the temperature inside waiting room 3...). The relationship between the individual quality and its (unique) bearer is the inherence: $\mathrm{I}(x, y)$ "the individual quality $x$ inheres to the entity $y$ ". The category $\mathrm{Q}$ is partitioned into several quality kinds $\mathrm{Q}_{i}$, for example, color, weight, temperature, the number of which may depend on the domain of application. Each quality kind $\mathrm{Q}_{i}$ is associated to (one or more) quality spaces $\mathrm{S}_{i, j}$ that provide a measure for the given quality. We say that individual qualities are located at a certain point of a space $S$ at time $t$ : $\mathrm{L}(x, y, t):$ : $x$ is the location of quality $y$ at time $t$ ".

Spaces allow for evaluating relationships between objects from the point of view of a given quality. For example, "the temperature inside room $3(q)$ is higher than the temperature inside room $4\left(q^{\prime}\right)$ " is represented in the ontology by assuming spaces of values with order relations and by saying that the location of the individual property $q$ is lower than the location of $q^{\prime}$. Spaces may be more structured objects and they may be specified along several dimensions ${ }^{1}$.

The axioms that define the relationships between individual qualities, locations, and spaces state for example that every individual quality must be located in some of its associated spaces and that the location in a particular space must be unique, cf. [4]. The category of regions $\mathrm{R}$ includes subcategories for spatial locations and a single region for time, denoted T: T $(x)$ means " $x$ is a time location" (e.g. October 10, 2012, 12:31 $\mathrm{PM})$. The relation $\operatorname{PRE}(x, t)$, where $t$ is a time location, allows to specify that " $x$ is present at time $t^{\prime \prime}$.

Arbitrary sums AS allow for talking about mereological sums of particulars. We shall apply sums directly when we will approach ontological entanglement.

The category of concepts is used in particular to model social objects. Concepts are reified properties that allow for viewing them as entities and to specify their attributes. In particular, concepts are used when the intensional aspects of a property are salient

\footnotetext{
${ }^{1}$ Quality spaces are related to the famous treatment of concepts in [15].
} 
for the modeling purposes. The relationship between a concept and the object that instantiates it is called classification $\operatorname{CF}(x, y, t)$ " $x$ is classified by concept $y$ at time $t$ ".

We represent the DOLCE taxonomy as a tree (cf. Figure 1, where we listed only the categories that are relevant for the current argument).

In the next paragraphs, we informally describe three modules: the physical, the mental, and the social module. We present them briefly, in order to use them to exemplify our definitions of entanglement.

\subsection{Physical module}

In order to simplify the presentation, we assume that the physical module includes physical objects, such as rocks, chairs, planets. Moreover, we include physical qualities (PQ), i.e. measurable qualities, such as weight, length, or temperature. A category of interest for the present discussion that we list among the subcategories of physical object is that of technical artifact, which includes tools, like for instance screwdrivers. Technical artifacts are of course non-reducible to mere physical objects, in the sense that they have some specific properties that characterize them for what they are that are evidently not physical. For instance, they have a specific function that has been attributed to them by intentional agents [3]. We shall come back to the discussion of artifacts as they provide an interesting general example of entanglement [5].

Other kinds of artifacts can also be viewed as exemplifications of entangled objects, like pieces of arts. Let's take a classical example in philosophy, that of a statue constituted by a lump of clay and its shape: while the clay certainly belongs to the physical realm, the shape could probably be seen as the result of the intentions of the sculptor. Modeling pieces of arts should thus rely both on the physical and on the mental module.

A further hint we would suggest is that, under constructivist perspectives, also physical qualities could be seen as entangled, given the dependance of their measurement on the attuning of the outcomes of apparatuses and the conventional ascription of values to such outcomes [7]. Under such perspective, modeling measurable qualities involve the physical and the social module. This is not the way in which physical qualities are currently characterized in DOLCE, our claim here is just that entanglement would be required in a constructivist ontology of qualities.

\subsection{Mental Module}

The mental module includes particulars that are in general ascribable to and dependent on specific individual agents. For instance, beliefs, desires, intentions belong to the mental module of DOLCE. In [12], they are all collected under the category of computed objects, to render the idea that they are indirect, depending on other mental objects, and that they are distinct from percepts, which depend on something "external", for instance the physical world, if we take a realist stance.

If we follow this line of thought, we can see that percepts belong to the mental realm, but also depend on the physical one, so in order to model them also the physical module is required. 


\subsection{Social module}

One predicate that is particularly important for modeling socio-technical systems is the classification predicate: $\mathrm{CF}(x, y, t)$, " $x$ is classified as $y$ at time $t$ ". By using CF, we can define a special type of social object, namely the notion of role, e.g. student, the president of the US . Roles are supposed to be contextual properties, which are characterised by anti-rigidity (AR) and foundational dependence (FD): roles are concepts that classify entities at a certain point in time, but not necessarily classify them in each moment or each possible world in which they are present (AR) and that require a level of definitional dependence on another property (FD). In this sense, roles are social objects as they are grounded in a sort of Searlian counts as.

For instance, someone who is a student at a certain point, not necessarily will be a student all throughout his/her life and there are possible worlds in which he/she is not a student; in order for someone to be classified as an employee, we need someone else who is classified as an employer.

Given these characteristics, roles are essential to model organizations, as they allow to talk about properties that an individual acquires by virtue of the fact that she/he is member of an organization or has some rights/duties connected with the role he/she is playing in that very moment. Moreover, roles may also classify aggregates of individuals: in this case, we can model specific types of roles (e.g. ORG and GRP) in order to model groups and organizations (cf. [6] and [25]). In DOLCE it is also possible to treat norms and plans, cf. [2].

\subsection{Classifying information into modules}

We have briefly described a number of modules that compose DOLCE. We want now to classify information according to the module it belongs to. We define the predicates PM, MM, SM, which stand for physical, mental, and social module respectively. We thus proceed to classify the elements of the ontology according to their module. For instance, by using the three modules we have specified in the previous section, we can group the particulars of the foundational ontology as follows. For the sake of example, we just propose the following very simple grouping.

$$
\begin{array}{ll}
\text { a1 } & \mathrm{PM}(x) \leftrightarrow \mathrm{PO}(x) \vee \mathrm{PQ}(x) \\
\text { a2 } & \mathrm{MM}(x) \leftrightarrow \mathrm{MO}(x) \\
\text { a3 } & \mathrm{SM}(x) \leftrightarrow \mathrm{SO}(x) \vee \mathrm{SC}(x)
\end{array}
$$

By the subsumption relation, we can infer for instance that every particular that is below the category of social object is within the social module. For instance, assume that customer is a role, that is, it belongs to $R L, R L$ (customer). Since $R L$ is included in SC, then also SC (customer). Thus, by axiom 3, customer is in the social module. ${ }^{2}$ Therefore, the particulars in the ontology can be simply classified according to their module. $^{3}$

\footnotetext{
${ }^{2}$ Note that, although we predicate on concepts, e.g. $\mathrm{RL}$ (customer), all the definitions are in first order logic. As usual in DOLCE, concepts are reified in order to describe them.

${ }^{3}$ In case we want to extend the classification into modules to propositional information concerning a specific domain, we need a little more caution. For instance, if we want to say that
} 


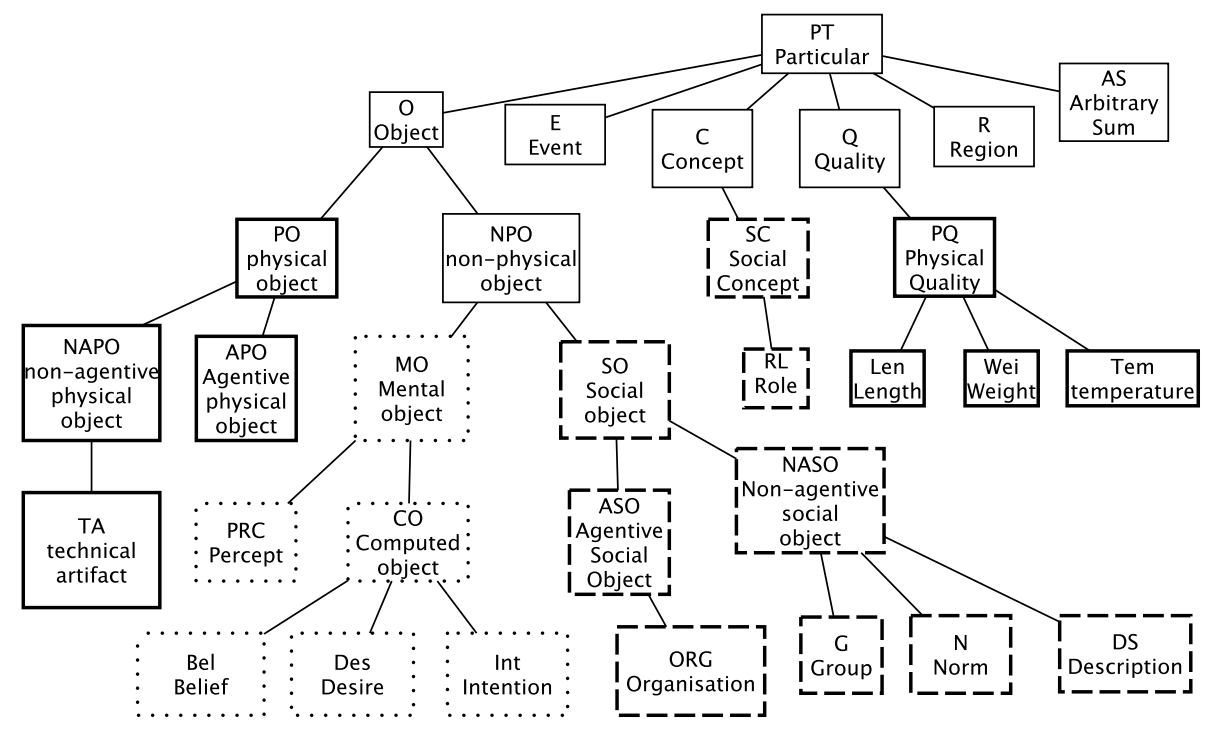

Fig. 1. An excerpt of the DOLCE Ontology. The thick lines indicate the physical module, the dotted lines indicate the mental module, and the dashed lines indicate the social module.

\section{A conceptualisation of entanglement}

We present now the formal treatment of entanglement from the perspective of critical realism, that is, by assuming that it is possible to provide a prior separation of the social and the material reality. The separation of social and material reality is reflected by the distinction of pieces of information into modules.

For simplifying our presentation, we restrict our formal description of entanglement to objects. We coin here the property of being entangled, or manifesting entanglement, we label it ent $(x)$, and we specify its different types.

a4 ent $(x) \rightarrow \mathrm{O}(x)$

In prinicple, other types of entities, such as concepts, properties, or events, may exhibit entanglement. We leave this for future work.

\subsection{Ontological entanglement}

A level of ontological entanglement seems to be supported by [1] in terms of "mutual constitution" of entangled entities. We approach the model of this mutual constitution

Mary is a customer at time $t$, we use the classification relation CF(Mary, customer, $\mathrm{t}$ ). Which is then the module of the proposition CF(Mary, customer, $t$ )? One way to cope with that is to extend the definition of modules to the predicates in DOLCE and assume that it is the predicate that determines the module. 
by means of the notion of grounding [14, 9, 20]. Grounding is a relation between particulars that is assumed to be a strict partial order (i.e. transitive and asymmetric). Here, we assume that grounding is defined on objects or on aggregates of objects. In [20], the notion of grounding is related to the possibility of distinguishing different ontological levels that arrange the particulars of an ontology into hierarchies.

We say that an object $y$ is grounded (existentially depends on) $x$ and write $x \prec y$ to express that the existence of $x$ grounds the existence of $y$. We view the concept of grounding as specifying the essential constituents of an object.

We are here assuming that an object may be grounded in the mereological sum of objects, denoted by $x+y .{ }^{4}$ For instance, a statue existentially depends on the mereological sum of the lump of clay and the shape that is given to it. The example of the statue guides our definition of ontological entanglement. We view a statue as showing the essential dependence on objects coming from different realms. That is, at any time, a statue cannot exist without its amount of matter and its given shape. Since amounts of matter and shapes belong to different modules, the statue suggests in its nature the entanglement of two objects coming from different realms. By generalizing this example, we define the ontological entanglement as follows:

d1 $\operatorname{ent}_{O}(x) \leftrightarrow \exists y, z\left(y+z \prec x \wedge \mathrm{M}(y) \wedge \mathrm{M}^{\prime}(z)\right)$

We assume that $\mathrm{M}$ and $\mathrm{M}$ ' label distinct modules. ${ }^{5}$ In words, definition (d1) means that an object $x$ manifests entanglement if it is grounded on two objects that belong to different modules.

For instance, one view of technical artifacts is that they are dependent on their physical substratum as well as on the intentional selection of the physical object with the purpose of attributing it a specific property, e.g. a rock becomes an artifact, a paperweight, by selecting it and attributing such function to it [3]. Thus, our approach formalizes the view of the dual nature of artifacts [18]. That is, a paperweight $p$ may be viewed as grounded on a physical object, the rock $r$, and on a mental object, the designer's goal $g: r+g \prec p$. Therefore, by axiom (d1), we can infer that a paperweight manifests entanglement.

On a closer inspection, the function of an artifact is not a mere mental object, that is, its function has to be sharable in principle by a community of users who acknowledge the physical object as a tool for a given purpose. By elaborating on this point, we can view artifacts as exhibiting entanglement between physical, mental, and social objects, i.e. they are also grounded on socially recognizable functions.

A further important example of ontological entanglement comes from social ontology. By endorsing a multiplicative view, we can see an organization as a distinguished object with respect to the aggregate of individuals that are members of the organization at a given time [25]. Therefore, if we assume that organizations exist as objects in our ontology, we may ask what are their constituents. In [25], we viewed organizations as grounded on aggregates of individuals as well as on normative constraints that bind and

\footnotetext{
${ }^{4}$ In [20], grounding is defined only for one-level objects, that is, objects that are on the same level of their parts. By assuming that a merelological sum such as $x+y$ may ground another object, we implicitly depart from [20] (in particular, we are rejecting axiom 14).

${ }^{5}$ We use $\mathrm{M}$ and $\mathrm{M}$ ' just as a shortcut to distinct modules.
} 
specify the organization. In this case, organizations exhibit entanglement of a physically dependent object, the aggregate of physical persons (in the category of AS), and the norms that are in place in the organization.

\subsection{Epistemological entanglement}

Epistemological entanglement concerns the possible ways in which an agent knows an object of the ontology. We model the epistemological relationship between an agent and an object by means of the relation of definition between a concept and a description. For the formal details, we refer to [22]. The main difference with [22] is that we apply descriptions to objects and not just to concepts. A description of an object is the way in which the agents may understand the object, may talk about it, may refer to it.

$$
\begin{array}{ll}
\text { a5 } & D S(x) \rightarrow N A S O(x) \\
\text { a6 } & U S(x, y) \rightarrow D S(y) \\
\text { a7 } & D F(x, y) \rightarrow(C(x) \vee O(x)) \wedge D S(y) \\
\text { a8 } & D F(x, y) \wedge D F(x, z) \rightarrow x=y
\end{array}
$$

Descriptions are non-agentive social objects, (a5). By making them social objects, we assume that their meaning is shared among agents. Thus, descriptions are not private mental entities, they are accessible by communities of agents. Axiom (a6) requires some explanation. Descriptions may only use concepts in [22]. In general, a description may use heterogenous particulars, such as other objects, events, properties, etc. For instance, I may define the number 2 by means of the description "the successor of 1 " that uses another object, i.e. the number 1, and a property, "being the successor of". We are not claiming that a description "contains" those particulars, we simply exploit the generality of the relation use in order to avoid a demanding characterization of descriptions. For instance, the description of a physical object may appeal to physical properties or to other physical objects. That does not mean that an abstract object such as the description contains physical objects. Moreover, a description of a social object such as a contract may use the individuals that are bounded by the contract, the roles that the individuals may play, the actions that each agent is bounded to perform, and so on. That is why in axiom (a6) we do not restrict the type of entities that may be used in a description. This is due to the quite abstract nature of $u s e .{ }^{6}$ By (a7), we say that a concept or an object may be defined by a description. For the sake of simplicity, we assume that there is only one description of an object, (a8). ${ }^{7}$ By assuming a single description of objects and concepts, we are implicitly assuming that descriptions completely specify their objects, that is, we are not including partial descriptions.

We are ready now to present the definition of epistemological entanglement.

$$
\text { d2 } \operatorname{ent}_{E}(x) \wedge D F(x, y) \rightarrow \exists v, w\left(U S(v, y) \wedge U S(w, y) \wedge \mathrm{M}(v) \wedge \mathbf{M}^{\prime}(w)\right)
$$

\footnotetext{
${ }^{6}$ One way to cope with this problem is to view descriptions as only composed by concepts and to assume that DOLCE contains concepts for any particular, i.e. concepts of individuals, physical objects, properties, $n$-ary relations, qualities, etc.

${ }^{7}$ We view this assumption as a mere simplification move. In general, one may think about many descriptions of the same objects and discuss whether they are equivalent.
} 
In words, an object manifests epistemological entanglement iff the description that defines it uses concepts (or objects, qualities, etc.) belonging to different modules. An example of epistemological entanglement is given by the objectification of a role. Assume that in our ontology roles, such as student, are distinct from the persons who are classified as students, that is roles are instantiated as particulars in the ontology. A description of a student then must refer to its physical substratum as well as to the normative features involved in the social concept of student.

It is possible to show that the ontological entanglement entails the epistemological one. Intuitively, if an object essentially depends on objects in different realms, then its definition should use elements coming from different modules. We can formalize this intuition by means of the following axioms.

a9 $\mathrm{M}(x) \wedge D F(x, d) \rightarrow \exists y(U S(y, d) \wedge \mathrm{M}(y))$

a10 $\quad x_{1}+\cdots+x_{m} \prec x \wedge D F(x, d) \wedge D F\left(x_{1}, d_{1}\right) \wedge \cdots \wedge D F\left(x_{m}, d_{m}\right) \wedge U S\left(y_{1}, d_{1}\right) \wedge$ $U S\left(y_{m}, d_{m}\right) \rightarrow U S\left(y_{1}, d\right) \wedge \cdots \wedge U S\left(y_{m}, d\right)$

Axiom (a9) states that if an object belongs to a certain realm, then its description must use at least one concept from that realm. For instance, a description of a physical object may refer to its weight, its colors, etc. This does not mean that the description must use only concepts from that realm. For instance, a description of my pen may be "the pen my father gave me for my birthday", that includes a physical property, i.e. being a pen, as well as social concepts, i.e. presents and birthday.

Axiom (a10) states that if an object $x$ is grounded on other objects $x_{1}, \ldots, x_{m}$ each defined by its respective description, then a description of $x$ must include elements from those descriptions. For instance, the description of a statue shall use concepts referring to its matter and to its shape. Axiom (a10) makes sense if we, as we do, assume complete descriptions. In case of partial descriptions, for instance, one may partially define a statue by mentioning its shape. Assuming the hypothesis that there is in our ontology a description of $x$, by (a9) and (a10), we can infer that:

$$
\mathbf{t 1} \quad \operatorname{ent}_{O}(x) \wedge \exists d D F(x, d) \rightarrow \operatorname{ent}_{E}(x)
$$

The assumption concerning the existence of a definition of $x$ is redundant if we assume that every object in the ontology must be defined by a description. ${ }^{8}$ That (t1) follows can be easily shown. Assume that $d$ defines $x$. Then, since $x$ is ontologically entangled, by definition (d1), $x$ is grounded on at least two objects $y$ and $z$ belonging to different modules. By axiom (a10), the description of $x$ must use elements from the descriptions of $y$ and $z$. By axiom (a9), such elements belong to different modules, so by definition (d2) we conclude. Therefore, if we assume that every object of the ontology is definable, ontological entanglement entails epistemological entanglement. Note that ( $\mathrm{t} 1$ ) also depends on the fact that we are excluding partial descriptions. In case our (unique) description of an entangled object is partial - for instance, it refers only

\footnotetext{
${ }^{8}$ Ontologies are here motivated by knowledge representation, thus it is reasonable to assume that every object has a description. However, it is up to the modeler whether to make this choice.
} 
to a specific realm - we would have ontologically entangled objects that do not exhibit epistemological entanglement.

Viceversa, we ask whether epistemological entanglement entails ontological entanglement. Formally this is not the case, as we have no means to infer that an object is grounded on other objects. We believe that this is conceptually correct and it may account for the following case. Suppose we do not want to make a distinction in our ontology between actions and events, that is, in our ontology, actions are of the same type as events. Even if it is so, actions can be conceptually separated from events because they have to be described as intentional. This is in a nutshell Davidson's point [10]. Hence, although we do not view actions as separated with respect to events - and accordingly we assume that actions do not exhibit ontological entanglement between a physical and a mental realm - any possible description of actions must include elements coming from the intentional, mental, or social module. Thus, actions do exhibit epistemological entanglement.

\section{Conclusion: Towards agential realism}

We have seen that by presupposing that information can be divided into modules, it is possible to provide an ontological model of entanglement as a property of objects: entangled objects are those that manifest properties belonging to different modules. We conclude by providing a few elements for approaching an agential realist position on the distinction between social and material. By rephrasing agential realism within our framework, there is no separation of objects into modules that is prior to the act of a knowing subject. Therefore, an ontological separation into modules appears illegitimate from the perspective of agential realism. On a closer inspection, however, there is indeed a way to express agential realism by means of DOLCE. One of the main motivations of DOLCE is to provide a clear representation of how a cognitive agent looks at the reality. Therefore, DOLCE can be viewed as the result of the categorization made by a knowing agent of a certain domain of interest. From this point of view, then, we can justify the separation of information into distinct modules of DOLCE as the result of an "agential cut" [1] that a knowing agent has performed on a domain of interest. The challenge of representing agential realism from the point of view of the ontological modeling is to capture and model how a knowing agent performs the categorization of reality into modules. That is, the challenge is to model the very notion of the agential cut.

The general idea of an ontological model of agential realism is based on an inverse process with respect to the one we have depicted in Section 3: instead of starting from an already given separation into modules and then defining entanglement, we need to start from a general notion of possibly entangled object and retrieve the modules as the agent's categorization choices.

We can use our previous definition of ontological entanglement (cf Definition (d1)) to give at least an idea of a possible modellization. The intuition leading our view is that information is going to be separated into modules, whenever an agent needs to express properties of an object that she/he is viewing as incompatible. If there is no object that may in principle share incompatible properties, there is no need for separating pieces of information into distinct modules. Thus, suppose that an agent is assuming that an ob- 
ject $a$ may have two incompatible properties $P(a)$ and $Q(a)$. The agent now can decide whether the incompatibility lies at an ontological or at an epistemological level, that is, whether $P$ and $Q$ represent incompatible constitutions of $a$ (ontological entanglement) or they are prone to represent incompatible descriptions of $a$ (epistemological entanglement). By using definitions (d1) or (d2), the agent is led to separate the properties $P$ and $Q$ by associating them to distinct modules. In the situation we have described, modules are not given, they are constructed starting from the agent's view of incompatibility of pieces of information. Building on that, we can also consider as an important challenge for multiagent systems the problem of coordinating different conceptualizations given by different agential cuts.

This simplified example of how to define modules as the outcome of an agential cut of reality poses a fundamental challenge in knowledge representation and ontology modeling and it is the aim of our future work. By taking seriously the motto "there is no social that is not also material, and no material that is not also social" [24, 1437], entanglement is no longer a property of some entity of the ontology, rather it becomes the designing principle for approaching the general construction of an ontology. According to this view, the task of a foundational ontology is not to separate the domain of discourse into rigid categories, relations, and modules that group agent-independent entities, it rather has to model the relationship between agents who focus on aspects of reality and the entities that are postulated or produced by the agents' activities. Accordingly, categories and modules become features of entities that may be intentionally selected, rather than rigid categorizations of the domain of discourse. In order to approach the development of such a view, that is a subject aware ontology, we need to embrace a constructivist view of entities and model their production. This is of course a long term plan, however we believe that it is worth pursuing.

\section{Bibliography}

[1] K. Barad. Meeting the Universe Halfway. Qunatum Physics and the Entanglement of Matter and Meaning. Duke University Press, 2007.

[2] G. Boella, L. Lesmo, and R. Damiano. On the ontological status of plans and norms. Artif. Intell. Law, 12(4):317-357, 2004.

[3] S. Borgo, M. Franssen, P. Garbacz, Y. Kitamura, R. Mizoguchi, and P. E. Vermaas. Technical artifacts: An integrated perspective. Applied Ontology, 9(3-4):217-235, 2014.

[4] S. Borgo and C. Masolo. Foundational choices in dolce. In S. Staab and R. Studer, editors, Handbook on Ontologies. Springer, second edition, 2009.

[5] S. Borgo, D. Porello, and N. Troquard. Logical operators for ontological modeling. In Formal Ontology in Information Systems - Proceedings of the Eighth International Conference, FOIS 2014, September, 22-25, 2014, Rio de Janeiro, Brazil, pages 23-36, 2014.

[6] E. Bottazzi and R. Ferrario. Preliminaries to a DOLCE ontology of organizations. Int. Jour. of Business Process Integration and Management, 4(4):225-238, 2009.

[7] E. Bottazzi, R. Ferrario, and C. Masolo. The mysterious appearance of objects. In M. Donnelly and G. Guizzardi, editors, FOIS 2012 - Formal Ontology in Information Systems, Seventh International Conference, pages 59 - 72, 2012. 
[8] W. J. CLANCEY, P. SACHS, M. SIERHUIS, and R. V. HOOF. Brahms: simulating practice for work systems design. International Journal of Human-Computer Studies, 49(6):831 865, 1998.

[9] F. Correia and B. Schnieder. Metaphysical grounding: Understanding the structure of reality. Cambridge University Press, 2012.

[10] D. Davidson. Essays on Actions and Events. Oxford University Press, 1980.

[11] F. E. Emery and E. Trist. Socio-technical systems. In Management Sciences: Models and Techniques, volume 2, pages 83-97. Pergamon, 1960.

[12] R. Ferrario and A. Oltramari. Towards a computational ontology of mind. In A. C. Varzi and L. Vieu, editors, Formal Ontology in Information Systems, Proceedigs of the Intl. Conf. FOIS 2004, pages 287-297. IOS Press, 2004.

[13] R. Ferrario and L. Prévot. Formal ontologies for communicating agents. Applied Ontology, 2(3):209-216, 2007.

[14] K. Fine. Guide to ground. Metaphysical grounding: Understanding the structure of reality, pages 37-80, 2012.

[15] P. Gärdenfors. Conceptual spaces - the geometry of thought. MIT Press, 2000.

[16] C. Ghidini and F. Giunchiglia. What is local models semantics? In P. Bouquet, L. Serafini, and R. H. Thomason, editors, Perspectives on Context, chapter 2, pages 19-42. CSLI Publications, 2008.

[17] C. Joslyn and L. M. Rocha. Towards semiotic agent-based models of socio-technical organizations. In IN: PROC. AI AND SIMULATION 2000, ED. HS SARJOUGHIAN ET AL, pages 70-79, 2000.

[18] P. Kroes. Engineering and the dual nature of technical artefacts. Cambridge journal of economics, page bep019, 2009.

[19] P. M. Leonardi. Theoretical foundations for the study of sociomateriality. Information and Organization, 23:59-76, 2013.

[20] C. Masolo. Understanding ontological levels. In Proc. of the 12th Int. Conf. on the Principles of Knowledge Representation and Reasoning (KR-2010), 2010.

[21] C. Masolo, S. Borgo, A. Gangemi, N. Guarino, and A. Oltramari. Wonderweb deliverable d18. Technical report, CNR, 2003.

[22] C. Masolo, L. Vieu, E. Bottazzi, C. Catenacci, R. Ferrario, A. Gangemi, and N. Guarino. Social roles and their descriptions. In Proc. of the 6th Int. Conf. on the Principles of Knowledge Representation and Reasoning (KR-2004), pages 267-277, 2004.

[23] J. McCarthy. Notes on formalizing context. In Proceedings of the 13th International Joint Conference on Artifical Intelligence - Volume 1, IJCAI'93, pages 555-560, San Francisco, CA, USA, 1993. Morgan Kaufmann Publishers Inc.

[24] W. J. Orlikowski. Sociomaterial practices: Exploring technology at work. Organization Studies, 28:1435-1448, 2007.

[25] D. Porello, E. Bottazzi, and R. Ferrario. The ontology of group agency. In Formal Ontology in Information Systems - Proceedings of the Eighth International Conference, FOIS 2014, September, 22-25, 2014, Rio de Janeiro, Brazil, pages 183-196, 2014.

[26] D. Porello, F. Setti, R. Ferrario, and M. Cristani. Multiagent socio-technical systems: An ontological approach. In Coordination, Organizations, Institutions, and Norms in Agent Systems IX - COIN 2013, pages 42-62, 2013. 